\title{
Nivolumab Versus Docetaxel for Previously Treated Advanced Non-Small Cell Lung Cancer in China: A Cost-Effectiveness Analysis
}

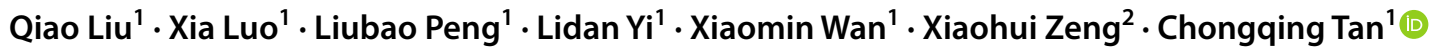

Published online: 2 November 2019

(c) The Author(s) 2019

\begin{abstract}
Background and Objective The economic assessment of immuno-oncology agents in Chinese patients is limited despite a need for new therapies. Nivolumab is the first immune checkpoint inhibitor approved for the second-line treatment of nonsmall cell lung cancer (NSCLC) in China, and it significantly prolongs overall survival. However, considering the high cost of nivolumab, it is urgent to assess its value in China in terms of both efficacy and cost. The objective of this study was to investigate the cost-effectiveness of nivolumab vs docetaxel in the second-line setting for NSCLC patients from the Chinese healthcare system perspective.

Methods A Markov model consisting of three health states, was designed to evaluate the lifetime cost and effectiveness of nivolumab vs docetaxel in the second-line treatment of NSCLC patients. Clinical data was derived from the CheckMate 078 phase III clinical trial, which included 504 patients predominantly from China. Parametric survival models to fit and extrapolate survival data were chosen based on clinical rationality, visual fit and statistical goodness-of-fit. Lifetime costs and health outcomes were calculated, and US $\$ 28,899$ and $\$ 63,564$ per quality-adjusted life-year (QALY) were selected as the willingness-to-pay (WTP) threshold values for general regions and affluent regions, respectively. One-way and probabilistic sensitivity analyses were undertaken to explore the robustness of the model. Additional subgroup analyses were performed. Results In base case analysis, Nivolumab yielded an additional 0.24 QALYs, at a cost of \$93,307 per QALY. Sensitivity analyses suggested that the results to be most sensitive to the price of nivolumab per kg (mean $\$ 60.00$; range $\$ 26.00-\$ 60.00$ ) and the mean patient weight ( $65 \mathrm{~kg}$, range $52-78 \mathrm{~kg}$ ). Utility values in progression-free survival state (mean 0.804 ; range $0.643-0.965)$ and overall survival hazard ratio $(0.68 ; 97.7 \%$ CI $0.52-0.90)$ had moderate impact on the model results. Subgroup analyses indicated that nivolumab was most cost-effective for patients who were 65 years of age or older ( $\$ 85,171 / \mathrm{QALY})$, followed by female patients ( $\$ 85,273 / \mathrm{QALY})$ and patients with tumor PD-L1 expression at least 1\% (\$90,309/QALY).

Conclusions Nivolumab is unlikely to be cost-effective compared with docetaxel for patients with previously treated advanced NSCLC in China. Ensuring that nivolumab is included in the National Reimbursement Drug List (NRDL) may be a valid mean of meeting extensive treatment demands in China.
\end{abstract}

Electronic supplementary material The online version of this article (https://doi.org/10.1007/s40261-019-00869-3) contains supplementary material, which is available to authorized users.

Xiaohui Zeng

zengxiaohui2008@csu.edu.cn

$\triangle$ Chongqing Tan

tanchongqing@csu.edu.cn

1 Department of Pharmacy, The Second Xiangya Hospital of Central South University, Changsha 410011, Hunan, People's Republic of China

2 PET-CT Center, The Second Xiangya Hospital of Central South University, Changsha 410011, Hunan, People's Republic of China

\section{Key points}

1. Nivolumab could prolong the progression-free survival, and was associated with higher costs and greater benefit (i.e. QALYs and LYs).

2. Nivolumab was most cost-effective for patients who were 65 years of age or older, followed by female patients and patients with tumor PD-L1 expression at least $1 \%$.

3. Nivolumab is unlikely to be cost-effective largely lay with its high price, but it could be negotiated by the National Healthcare Security Administration (NHSA) to reduce the price given the unmet clinical need. 


\section{Introduction}

Lung cancer remains the most prevalent cancer and the leading cause of cancer-related death in China, with 733,000 newly diagnosed cases and 610,000 deaths in 2015 [1]. Approximately $85 \%$ (range $80 \%$ and $90 \%$ ) of cases are represented by non-small cell lung cancer (NSCLC) [2-4], and majority of these cases are advanced disease at the time of first diagnosis [5]. Historically, the treatment for advanced NSCLC (aNSCLC) is rarely curative, and usually has a poor effect on increasing survival [3], with a 5-year survival rate of $16 \%$ [6]. Despite significant advances in targeted therapies, antiangiogenics, and immunotherapy for the first-line treatment of aNSCLC [7-9], subsequent lines of therapy for patients who experience progression on/after first-line chemotherapy are generally limited [10].

In the last 10 years, the introduction of immune checkpoint inhibitors (ICIs) into the second-line setting for NSCLC, particularly inhibitors of the programmed cell death protein 1 (PD-1) axis, has transformed the therapeutic landscape in this recalcitrant disease [11]. In China, nivolumab is the first PD-1 inhibitor licensed as a subsequent agent for aNSCLC. The pivotal studies (CheckMate 017 and CheckMate 057 Phase III trials, CA209-003 Phase I study) [12, 13], confirmed that nivolumab dramatically prolonged overall survival (OS), and had a more favorable safety profile compared with standard second-line treatment, docetaxel, in patients with pre-treated aNSCLC. However, these trials enrolled patients primarily from North America and Europe, and included very few Asian patients. It is noteworthy that the CheckMate 078 clinical trial, at the recent data cutoff (October 27, 2017), was the first Phase III trial to compare the efficacy and safety of nivolumab versus docetaxel in a predominantly Chinese population of patients $(N=504)$ with pre-treated aNSCLC [14], a statistically significant and clinically meaningful improvement in OS was found for patients assigned to nivolumab compared with docetaxel (12.0 vs 9.6 months, hazard ratio [HR] $0.68 ; 97.7 \%$ confidence interval [CI] 0.52-0.90; $p=0.0006)$. The significant superiority with regard to OS was consistent across most prespecified subgroups, including histology and tumor PD-L1 expression level subgroups. Furthermore, nivolumab was well-tolerated, with a lower incidence of grade $\geq 3$ treatment-related adverse events versus docetaxel (10\% vs $48 \%$ ).

Despite its remarkable clinical benefit and lower risk over conventional chemotherapy, nivolumab has induced high cost. Since 2017 the National Healthcare Security Administration (NHSA) has held a series of negotiations with pharmaceutical companies regarding oncology drugs, and many cancer drugs have been included in National Reimbursement Drug list (NRDL) with the price falling by an average of $56.7 \%$ [15]. Unfortunately, due to the fact that nivolumab has not been approved by the China Food and Drug Administration (CFDA) until June 2018, it was not presented in those negotiations and little is known about its cost-effectiveness for Chinese NSCLC patients.

Therefore, the present study aimed to evaluate cost and effectiveness of nivolumab versus docetaxel for aNSCLC patients after prior systemic chemotherapy from the perspective of Chinese healthcare system. Information on the cost effectiveness of nivolumab is required by healthcare decision makers to determine the relative role of this novel treatments in aNSCLC.

\section{Methods}

\subsection{Analytical Overview and Model Structure}

A Markov model was developed to evaluate the long-term costs and effectiveness associated with two second-line treatment strategies for patients with aNSCLC (Fig. 1): (1) nivolumab $3 \mathrm{mg} / \mathrm{kg}$ every 2 weeks; (2) docetaxel $75 \mathrm{mg} / \mathrm{m}^{2}$

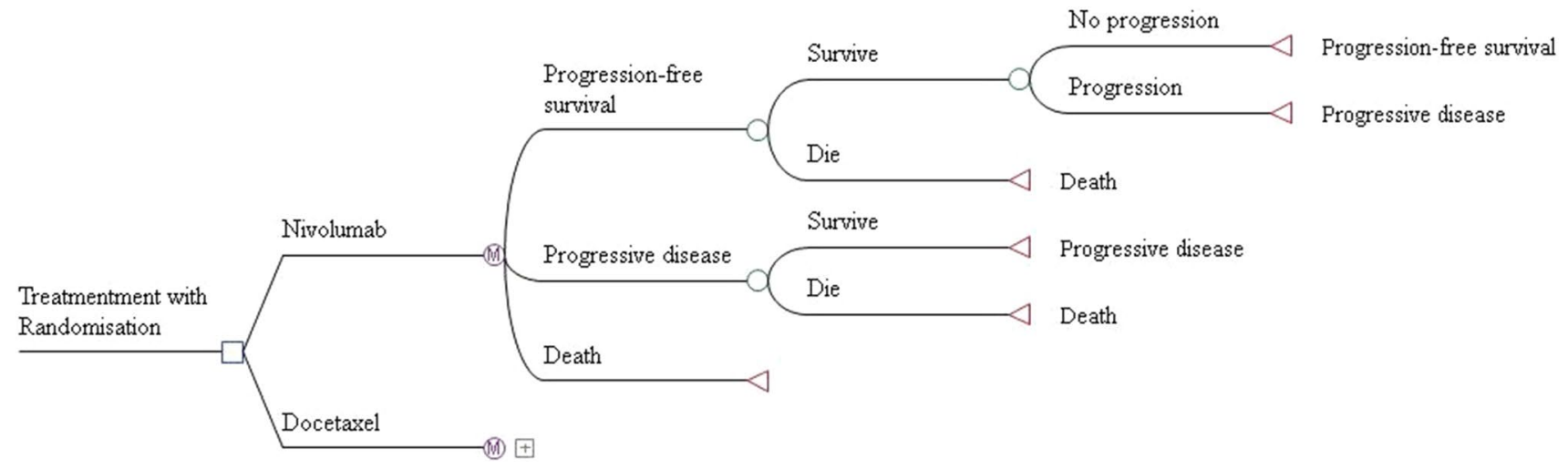

Fig. 1 Markov model structure of nivolumab and docetaxel strategies for the treatment of patients with previously treated advanced non-small cell lung cancer (NSCLC) 
every 3 weeks. To maintain consistency with the CheckMate 078 trial, a hypothetical cohort with confirmed stage IIIB or IV or recurrent squamous or non-squamous NSCLC patients progressing on/after one previous platinum-based doublet chemotherapy, and negative for EGFR mutation or ALK translocation was created to compare these two treatments, and the starting age of patients was assumed to be 60 years in this model. This economic analysis was based on a clinical trial and model techniques, and it did not require the approval of the institutional research ethics board.

Three mutually exclusive health states were included in the model to simulate aNSCLC progression: progressionfree survival (PFS) state, progression survival (PS) state and death. The Markov cycle length was 3 weeks, and the time horizon was lifetime. All patients were in the PFS state initially and were randomly treated with nivolumab or docetaxel. During each Markov cycle, the patients may remain in PFS state or transition to PS state or death. We assumed that the second-line treatments continued until disease progression or unacceptable toxicity without switching between the two treatments, and then both arms could receive subsequent treatments until death. As reported in the CheckMate 078 trial, $42 \%$ of patients in both arms received subsequent systemic therapy after disease progressed. The remaining patients would receive the best supportive care (BSC) directly on the basis of current clinical guidelines in China [15].

The primary outcomes in the model were measured in terms of the total cost, life years (LYs), quality-adjusted life-years (QALYs), and incremental cost-effectiveness ratios (ICERs). Cost was estimated from the perspective of the Chinese healthcare system and only direct medical care costs were considered in this model. We adopted a half-cycle correction in the model. Both costs and outcomes were discounted at an annual rate of $3 \%$. Due to the unbalanced economic development in China, we selected \$28,899/QALY as the willingness-to-pay (WTP) threshold value for general regions and \$63,564/QALY for affluent regions [16, 17].

\subsection{Model Survival and Progression Risk Estimates}

Kaplan-Meier survival data for OS and PFS were available from the CheckMate 078 trial at the updated data cutoff (October 27, 2017). The probability of death in the docetaxel arm was estimated based on OS curve associated with docetaxel treatment. GetData Graph Digitizer software package (version 2.25; http://www.getdata-graphdigitizer.com/index .php) was used to extract the data points from the OS curve, which were then used to fit parametric survival models. The PFS probability for docetaxel arm was obtained by the same approach. To improve the accuracy of the model, we generated the pseudo-individual patient data following the methodology of Hoyle [18]. According to clinical rationality, visual fit and statistical goodness-of-fit test, using Bayesian information criterion (BIC) and Akaike's information criterion (AIC), we found Weibull distribution provided the most appropriate fit to pseudo-individual patient data among the exponential, log-logistic, and log-normal distributions (see Supplementary Material Appendix 1). The Weibull parameters of the docetaxel group, scale $(\lambda)$ and shape $(\gamma)$ were estimated by R software (version 3.3.1, http://www.r-proje ct.org).

For the nivolumab arm, the Weibull parameters were adjusted by the HR for PFS and OS reported in the CheckMate 078 trial, that is, the shape $(\gamma)$ parameters of the nivolumab arm were the same as those of the docetaxel $\operatorname{arm}\left(\gamma_{\text {Nivolumab }}=\gamma_{\text {Docetaxel }}\right)$, and the scale $(\lambda)$ parameters were multiplied by those of the docetaxel arm and the HR $\left(\lambda_{\text {Nivolumab }}=\mathrm{HR} \times \lambda_{\text {Docetaxel }}\right)$. Table 1 shows the final estimated Weibull parameters. The time-dependency transition probabilities in each Markov cycle were calculated based on the following formula:

$t p\left(t_{u}\right)=1-\exp \left\{\lambda(t-u)^{\gamma}-\lambda t^{\gamma}\right\}(\lambda>0, \gamma>0)$,

where $u$ is the Markov cycle and $t_{u}$ represents the arrival at state $t$ after $u$ Markov cycles [19].

\subsection{Utility Estimates}

QALYs in the model were weighted by survival time and health state utility value. We captured utilities for patients with NSCLC from the published study by Nafees et al., who assessed 23 states using the cognitive debrief content validation interviews with oncologists in 6 countries including China [20]. The utility values of 0.804 (0.536-0.883) and $0.321(0.05-0.473)$ were used for the PFS and PS state, respectively (Table 1). As reported in this literature, decreases in utility were found to be associated with rash, fatigue, neutropenia and hair loss. Accordingly, the utility values for PFS were estimated weighted by the proportion of the adverse events published in the CheckMate 078 trial [14], and the utility values were as follows: PFS (0.804), PFS plus rash (0.705), PFS plus fatigue (0.736), PFS plus neutropenia (0.604) and PFS hair loss (0.746) [20].

\subsection{Cost Estimates}

Direct medical cost included the cost of the drug, the management of adverse effects (AEs), routine follow-up in PFS state, subsequent systemic therapy in PS sate, BSC, and terminal-phase treatment (Table 1). We assumed a base-case patient with an average weight of $65 \mathrm{~kg}$ and a body surface area of $1.72 \mathrm{~m}^{2}$ [21]. The unit cost of nivolumab $(4.5 \mathrm{mg} /$ $\mathrm{kg}$ per cycle) was calculated based on the latest retail price. The unit cost of docetaxel $\left(75 \mathrm{mg} / \mathrm{m}^{2}\right.$ per cycle) was estimated according to the average prices of each specification 
Table 1 Base cases, ranges and distributional assumptions of parameters

\begin{tabular}{|c|c|c|c|c|}
\hline Parameters & Base case & Range & Distribution & Source \\
\hline \multicolumn{5}{|l|}{ Costs (US\$) } \\
\hline Nivolumab (4.5 mg/kg per unit) & 60.0 & $26.0-60.0$ & Fixed in PSA & Local charge \\
\hline Docetaxel (129 mg per unit) & 193.0 & $154.4-231.6$ & Fixed in PSA & Local charge \\
\hline Routine follow-up per unit ${ }^{\mathrm{a}}$ & 55.6 & $41.7-69.4$ & Lognormal & [26] \\
\hline $\begin{array}{l}\text { Subsequent systemic therapy in PS state per } \\
\text { unit }^{\mathrm{b}}\end{array}$ & 854.1 & $706.5-992.4$ & Lognormal & {$[25]$} \\
\hline BSC per unit ${ }^{\mathrm{c}}$ & 337.5 & $158.7-793.7$ & Lognormal & {$[26]$} \\
\hline Terminal phase cost per unit ${ }^{\mathrm{d}}$ & 2627.8 & $2291.8-2966.6$ & Lognormal & {$[25]$} \\
\hline Neutropenia per event & 461.5 & $415.4-507.7$ & Lognormal & {$[23]$} \\
\hline Anemia per event & 531.7 & $478.5-584.9$ & Lognormal & {$[23]$} \\
\hline Fatigue per event & 115.4 & $103.8-126.9$ & Lognormal & {$[23]$} \\
\hline Rash per event & 5.5 & $4.4-6.6$ & Lognormal & {$[24]$} \\
\hline \multicolumn{5}{|l|}{ Risk for treatment-related AEs } \\
\hline Neutropenia in nivolumab arm & 0.020 & $0.016-0.024$ & Beta & [14] \\
\hline Neutropenia in docetaxel arm & 0.200 & $0.160-0.240$ & Beta & [14] \\
\hline Anemia in nivolumab arm & 0.040 & $0.032-0.048$ & Beta & [14] \\
\hline Anemia in docetaxel arm & 0.260 & $0.208-0.312$ & Beta & [14] \\
\hline Fatigue in nivolumab arm & 0.100 & $0.008-0.120$ & Beta & [14] \\
\hline Fatigue in docetaxel arm & 0.250 & $0.200-0.300$ & Beta & {$[14]$} \\
\hline Rash in nivolumab arm & 0.120 & $0.096-0.144$ & Beta & [14] \\
\hline Rash in docetaxel arm & 0.030 & $0.024-0.036$ & Beta & [14] \\
\hline Hair loss in nivolumab arm & 0 & - & - & [14] \\
\hline Hair loss in docetaxel arm & 0.220 & $0.176-0.264$ & Beta & {$[14]$} \\
\hline \multicolumn{5}{|l|}{ Utilities } \\
\hline PFS & 0.804 & $0.643-0.965$ & Beta & {$[20]$} \\
\hline PS & 0.321 & $0.257-0.385$ & Beta & {$[20]$} \\
\hline PFS plus rash & 0.705 & $0.564-0.846$ & Beta & {$[20]$} \\
\hline PFS plus fatigue & 0.736 & $0.589-0.883$ & Beta & {$[20]$} \\
\hline PFS plus neutropenia & 0.604 & $0.483-0.725$ & Beta & {$[20]$} \\
\hline PFS plus hair loss & 0.746 & $0.597-0.895$ & Beta & {$[20]$} \\
\hline \multicolumn{5}{|l|}{ Parametric distribution } \\
\hline Docetaxel, OS, scale (Weibull) & 0.04848005 & - & Fixed in PSA & Estimated \\
\hline Docetaxel, OS, shape (Weibull) & 1.252947 & - & Fixed in PSA & Estimated \\
\hline Docetaxel, PFS, scale (Weibull) & 0.03381398 & - & Fixed in PSA & Estimated \\
\hline Docetaxel, PFS, shape (Weibull) & 1.207347 & - & Fixed in PSA & Estimated \\
\hline \multicolumn{5}{|l|}{ HR } \\
\hline HR of OS & 0.68 & $0.52-0.90$ & Lognormal & {$[14]$} \\
\hline HR of PFS & 0.77 & $0.62-0.95$ & Lognormal & {$[14]$} \\
\hline Discount rate $(\%)$ & 3 & $0-8$ & Fixed in PSA & {$[27]$} \\
\hline Patient weight (kg) & 65 & $52-78$ & Fixed in PSA & {$[21]$} \\
\hline
\end{tabular}

AEs adverse effects, $B S C$ best supportive care, $H R$ hazard ratio, $P F S$ progression-free survival, $P S$ progression survival, $O S$ overall survival

${ }^{a}$ The cost of routine follow-up included the cost of outpatient physician visit, hospitalization, and laboratory tests

${ }^{\mathrm{b}}$ Subsequent systemic therapy after PS included immunotherapy, targeted therapy and chemotherapy

${ }^{c}$ BSC referred to the intervention of clinical symptoms caused by cancer, including anti-inflammatory treatment, analgesic treatment, antiemetic treatment, thoracic and abdominal puncture decompression, blood transfusion and nutritional support

${ }^{\mathrm{d}}$ The terminal phase cost referred to the cost of palliative end-of-life 
weighted by its market share in 2018, which were obtained from a published study [22], and then was adjusted on the basis of the portion paid by basic medical insurance.

All treatment-related AEs reported in $\geq 10 \%$ of patients in the CheckMate 078 trial, including rash, fatigue, decreased appetite, anemia, leukopenia, neutropenia and alopecia, were considered in the model, because of the significant differences in incidence between the two groups. Based on the local oncologists' opinions and clinical practices, decreased appetite and alopecia does not require additional treatment, and the treatment for neutropenia also treats leukopenia. Therefore, the costs of those AEs were excluded from the total costs. AEs costs were derived from previous studies [23, 24].

Other costs were obtained from previously published studies [25, 26]. All costs are reported in 2018 US dollars. Considering that the Chinese medical costs are stable under central control by the government, the costs from different base years were not inflated.

\subsection{Sensitivity Analysis}

One-way sensitivity analyses (OSA) were carried out by all parameters that independently varied over a plausible range (displayed in Table 1), while other parameters were fixed to explore how the results were influenced by the given parameters and evaluate the robustness of the model. The ranges of parameters were mainly obtained from published studies, or by assuming a variance of $20 \%$ from the base-case value. As recommended by Chinese guidelines for Pharmacoeconomic evaluation, the discount rate was varied from 0 to $8 \%$ [27]. Given a likely scenario that nivolumab will be included in NRDL, the analysis was conducted based on the original price and the price discounted by $56.7 \%$, which was the average price reduction through NRDL negotiation. The result was visualized by a tornado diagram.

For the Monte Carlo simulation probabilistic sensitivity analysis (PSA), we performed 10,000 iterations by varying all parameters simultaneously to account for the uncertainty in model parameters. These parameters were set to follow a specific statistical distribution, beta distribution was used for utilities and risks, while log-normal distribution was selected for costs and HRs. The PSA results were showed by Cost-effectiveness acceptability curves (CEAC). The baseline values, ranges, and distributions of the model parameters are listed in Table 1.

Furthermore, we performed analysis for all the patient subgroups in the CheckMate 078 trial. Because of the inadequacy of data, we assumed the same data in all subgroups except for the OS HRs and PFS HRs. These analyses helped to speculate about the potential population of NSCLC patients who were most likely to benefit from nivolumab.
The subgroup-specific OS HRs and PFS HRs are listed in Supplementary Material Appendix 2.

\section{Results}

\subsection{Base-Case Results}

The estimated mean PFS time and life expectancy for patients receiving nivolumab was $0.37 \mathrm{LYs}$ and $1.19 \mathrm{LYs}$, respectively, an increase of $0.3 \mathrm{LYs}$ when compared with those for patients receiving docetaxel. Accounting for the quality of life, the patients treated with nivolumab were associated with higher costs $(\$ 40,599$ vs $\$ 18,378)$ and QALYs ( 0.55 vs 0.31 ) when compared to docetaxel. The model indicated that the ICER per LY gained and per QALY gained for nivolumab versus docetaxel were $\$ 74,126$ and $\$ 93,307$, respectively. It was observed that the benefit associated with nivolumab depended mainly on the prolongation of PFS, accordingly, the costs in the PFS state were much higher in the nivolumab arm than that in docetaxel arm (Table 2).

\subsection{One-Way Sensitivity Analysis}

The results of one-way sensitivity analysis were visualized by a tornado diagram (Fig. 2). The most sensitive parameters with the greatest influence on the ICER were the price of nivolumab and patient weight, when the price of nivolumab

Table 2 Summary of cost (US dollars) and outcome results in the base-case analysis

\begin{tabular}{llll}
\hline Item & Nivolumab & Docetaxel & Difference \\
\hline Mean LYs & & & \\
PFS state & 0.37 & 0.07 & 0.30 \\
PS state & 0.82 & 0.82 & 0.00 \\
Total & 1.19 & 0.89 & 0.30 \\
Mean QALYs & & & \\
PFS state & 0.29 & 0.05 & 0.24 \\
PS state & 0.26 & 0.26 & 0.00 \\
Total & 0.55 & 0.31 & 0.24 \\
Cost (US\$) & & & \\
PFS state & 22,707 & 410 & 22,297 \\
PS state & 16,622 & 16,646 & -24 \\
Dead state & 1270 & 1282 & -12 \\
Total & 40,599 & 18,338 & 22,261 \\
ICER (\$) & & & 74,126 \\
Per LY & & & 93,307 \\
Per QALY & & & \\
\hline
\end{tabular}

ICER incremental cost-effectiveness ratio, $L Y$ life-year, $P F S$ progression-free survival, $P S$ progression survival, $Q A L Y$ quality-adjusted life-year 
Fig. 2 Tornado diagram of the one-way sensitivity analysis revealing variables' influence on the ICER. The black dotted line represents the ICER of US\$93,305 per QALY from the base-case results, the black solid line represents the WTP of \$63,564/QALY for affluent regions in China. ICER incremental cost-effectiveness ratio, $Q A L Y$ quality-adjusted life-year

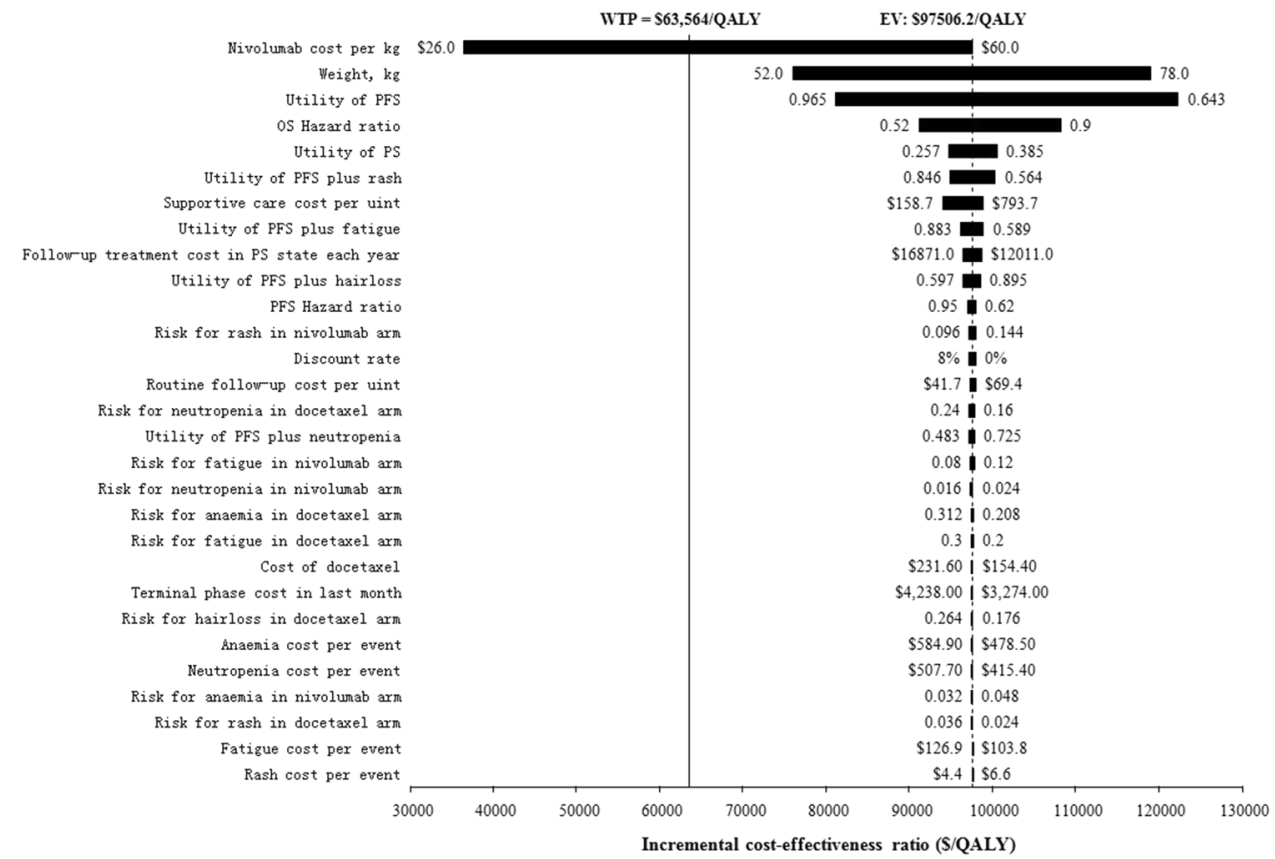

was $31.6 \%$ lower than its original price, the ICER would be under the WTP threshold of \$63,564/QALY for affluent regions. The other sensitive parameters included the utility of PFS, OS HR and utility of PS. The analyses suggested that all parameters in defined ranges except the price of nivolumab, failed to make ICER lower than the WTP threshold selected for affluent regions, and none of those parameters led to an ICER entrancing the WTP threshold selected for general regions.

\subsection{Probabilistic Sensitivity Analysis}

Figure 3 a presents the results of PSA using the cost-effectiveness acceptability curve (CEAC). The probability of nivolumab being cost effective compared with docetaxel was $4.6 \%$ at the WTP threshold of $\$ 63,564$ per QALY, while it is a zero probability at the WTP threshold of $\$ 28,899$ per QALY. The cost-effectiveness of nivolumab was likely sensitive to the thresholds of WTP, when the WTP threshold was $>\$ 40,000$, cases began to be cost-effective, and almost all cases achieved cost effectiveness when the WTP threshold reached $>\$ 320,000$. Figure $3 b$ demonstrates that the likelihood of nivolumab being cost-effective increased with decreasing the cost of nivolumab, when the price of nivolumab decreased by $30 \%$ and $70 \%$, the proportions of simulations with cost-effectiveness for nivolumab were $6.1 \%$ and $87.3 \%$, respectively, at the WTP of $\$ 63,564$ per QALY.

In addition, the results of the subgroup analyses revealed that nivolumab was most cost effective for patients who were aged $\geq 65$ years, followed by female patients and patients with tumor PD-L1 expression at least $1 \%$, squamous NSCLC (Supplementary Material Appendix 2).

\section{Discussion}

Nivolumab is the first PD-1 inhibitor proven to have a survival benefit for Chinese patients with aNSCLC, and was approved by China Food and Drug Administration (CFDA) in June 2018 [14]. However, as this high priced (\$60 per $4.5 \mathrm{mg}$ ) new drug has not yet been included in the NRDL, it is urgent to assess its value in terms of both efficacy and cost.

For patients with previously treated aNSCLC, the modelbased costs and benefits (i.e. QALYs and LYs) for nivolumab arm were greater than docetaxel arm, the corresponding ICERs were ranged from $\$ 85,171$ to 143,663 per QALY gained. Unfortunately, from the Chinese healthcare system perspective, nivolumab is unlikely to be cost-effective versus the standard second-line treatment docetaxel. The sensitivity analyses suggested a high likelihood of nivolumab being cost effective with increasing thresholds of WTP, which was consistent with previous study by Zeng et al. [19]. Due to the unbalanced economic development in China, the per capita gross domestic product (GDP) among province-level administrative units differs significantly. For example, it ranged from $\$ 4735$ in Gansu Province to $\$ 21,188$ in Beijing city in 2018, therefore, the WTPs were set for general regions (\$28,899, $3 \times$ per-capita GDP in 2018) and affluent regions $(\$ 63,546,3 \times$ per-capita GDP of Beijing) in this study. 

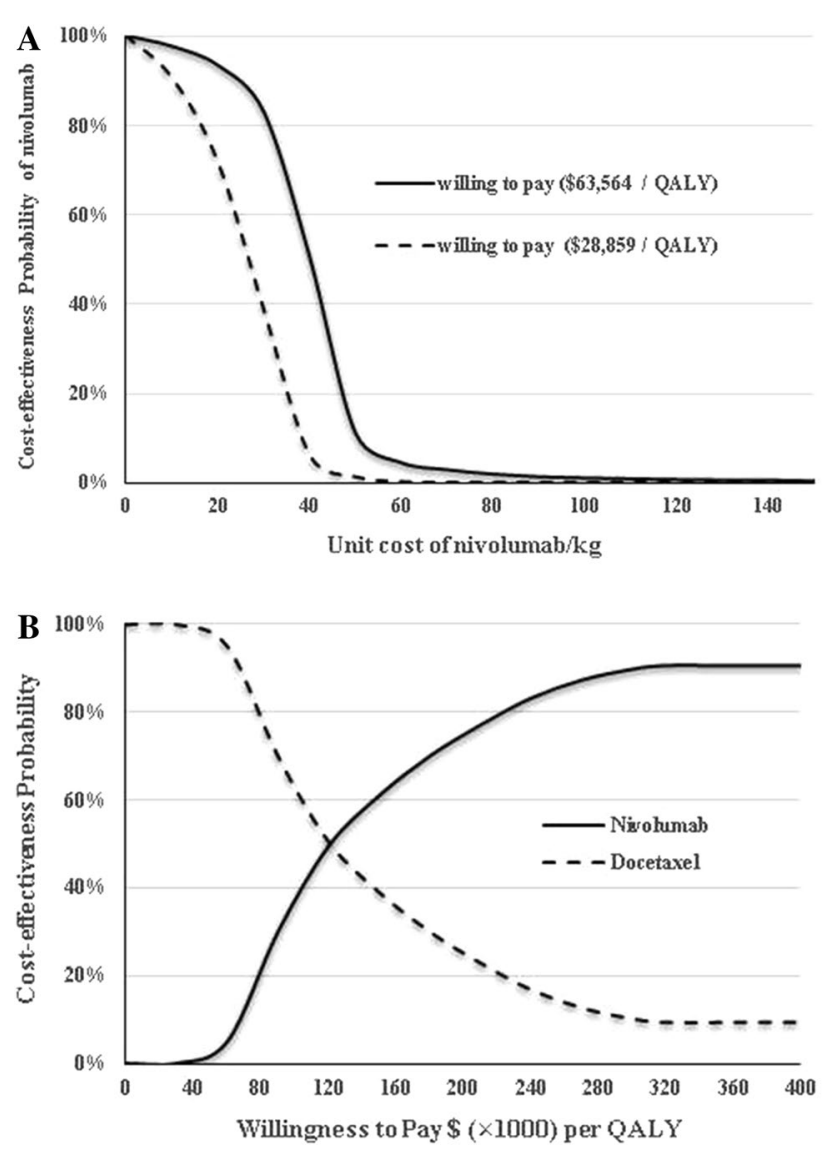

Fig. 3 a The cost-effectiveness acceptability curve. $Q A L Y$ qualityadjusted life-year. b Probability of cost-effectiveness of nivolumab versus docetaxel at different drug costs of nivolumab. Blue dotted lines represent the WTP threshold of US\$63,564/QALY for affluent regions, orange dotted lines represent the WTP threshold of $\$ 28,899$ / QALY for general regions and \$63,564/QALY. QALY qualityadjusted life years, $W T P$ willingness-to-pay

An important assumption made in discrete-time Markov models is that all state transitions occur simultaneously, at the end of each cycle. In reality, however, most kinds of transitions typically occur gradually throughout a time interval (on average, half-way through). This assumption does not affect reported probabilities (or the survival curve), but it may result in overestimation of expected survival in most models. For minimizing the influence on results, a half-cycle correction was adopted in current study.

The most influential parameters in our model were the price of nivolumab and patient weight, both of which were associated with the cost of nivolumab. From the perspective of cancer patients, the use of high-priced new drugs might impose a heavy financial burden, which likely leads to delay, abandonment, and discontinuation of treatment [28]. According to the sensitivity analysis, we found that when the price of nivolumab was discounted by more than $30 \%$, the ICER was lower than the WTP of $\$ 63,564 /$ QALY, and nivolumab therapy began to be cost-effective in affluent regions. Fortunately, a number of oncology drugs have recently been included in the NRDL negotiation conducted by the Chinese government, with price decreases ranging from 30 to $70 \%$. Therefore, negotiating nivolumab might be an effective way to make nivolumab less costly and more widely used in China.

It should be noted that, the utility of PFS played an important role in the model, and similar findings have been reported in previous studies [19, 29]. A new study on the utilities of NSCLC from six countries including China conducted by Nafees et al, showed that some common toxicities associated with treatment probably lead to a significant decline in the utility of PFS [20]. Toxicities including rash, fatigue, neutropenia and hair loss were observed in the CheckMate 078 trial [14]. For higher accuracy, we weighted the utility of PFS according to the risks of these toxicities.

Other sensitivity parameters were found in our model, such as OS HR, and PFS HR, which were used to adjust the Weibull survival curve for the nivolumab arm. Moreover, we applied suitable HRs to determine whether the cost effectiveness of nivolumab varies in subgroups. The results indicated that nivolumab therapy for patients who were aged $\geq 65$ years, female patients, and patients with $\geq 1 \%$ tumor PD-L1 expression resulted in lower ICERs $(\$ 85,171$ / QALY, \$85,273/QALY and \$90,309/QALY), which were strikingly consistent with previous study by Wan et al. [30]. Thus, from a more far-sighted perspective, age stratification, tumor typing and PD-L1 assays before treatment would be helpful to use nivolumab more cost-effectively.

To our knowledge, there were only two published economic evaluations of nivolumab versus docetaxel as second-line treatments for aNSCLC, one from an Australian healthcare system perspective and the other from a broader societal perspective in Canada [31, 32]. Both studies found that nivolumab was associated with substantial survival benefits at incremental cost, which was confirmed by our study. In addition, there is a similar economic study (conference abstract) from the Chinese healthcare payer's perspective. The major finding was that patients receive nivolumab resulting in an ICER of \$35,663 and \$25,118 per QALY compared with docetaxel in squamous and non-squamous aNSCLC, respectively [33]. The estimation of ICERs in our study was higher than that in Hu et al's study, which mostly resulted from the different source of clinical data. Moreover, the sensitivity analysis in Hu et al.'s study found that the drug costs were a key model parameter, which is completely consistent with our results. However, it is noteworthy that we have further discussed the current findings on the basis of the National Reimbursement Drug List (NRDL) negotiation, which may be of great benefit to healthcare decision-makers in China. 
Although other ICIs, such as pembrolizumab and atezolizumab, have recently been approved by the US Food and Drug Administration (FDA) as second-line settings for NSCLC patients [34], we have not evaluated these therapies because of the lack of robust head-to-head trials, and the fact that they are not yet available in China. Most notably, atezolizumab has been confirmed to have similar efficacy and applicability to nivolumab based on the results of the Phase 2 POPLAR trial [34]. In view of the largest population of lung cancer patients in China, further cost-effectiveness analyses are urgently needed to compare these innovative therapies from the perspective of China's healthcare system.

\section{Limitations}

There are several limitations in the present study. First, we acknowledge that the CheckMate 078 trial is a large randomized Phase III trial and precisely designed to provide the most exhaustive evidence to clinical date, Even more remarkably, it is the only trial that compared nivolumab with docetaxel in a predominantly Chinese patient population with pre-treated aNSCLC, but our model depends largely on the validity and generalizability of this trial, and any biases within the trial will inevitably affect our model. Second, the unit costs of healthcare were pooled across various sources. We conducted a series of sensitivity analyses by varying the costs within a wide range and found a minimal impact on the ICER for both arms. Third, due to the lack of sufficient data in the subgroups, the results of the subgroup analyses in our study should be carefully interpreted, given that the assumption of the consistency of clinical data between subgroups and overall patients. Fourth, on the basis of the visual fit and statistical goodness-of-fit, the long-term survival of nivolumab was extrapolated from the clinical data of the CheckMate 078 trial, which would likely overestimate OS in the long term. Although there was a slight numerical difference in costs and effectiveness between the long-term and 21.2 months, which was the maximum follow-up time in the CheckMate 078 trial, differences with regard to model result were not significant, nevertheless, when mature OS data are available, it will be essential to further confirm the current findings.

\section{Conclusions}

From the perspective of Chinese healthcare system, nivolumab was unable to be cost-effective versus the standard second-line docetaxel for patients with previously treated aNSCLC. When nivolumab is included in the NRDL, and the price of nivolumab discount exceeds $31.6 \%$, nivolumab therapy will be cost-effective in China.
Acknowledgements I certify that no individuals other than the listed co-authors contributed to this publication.

\section{Compliance with Ethical Standards}

Funding This work was supported by the Provincial Natural Science Foundation [Grant numbers 2019JJ50864]; the National Natural Science Foundation of China [Grant numbers 81401547, 81603081]; and the Key Science-Technology Research and Development Program of Hunan Province [Grant number 2016JC2062].

Conflict of interest Qiao Liu, Xia Luo, LiubaoPeng, LidanYi, Xiaomin Wan, Xiaohui Zeng and Chongqing Tan declare that they have no conflict of interest.

Open Access This article is distributed under the terms of the Creative Commons Attribution-NonCommercial 4.0 International License (http://creativecommons.org/licenses/by-nc/4.0/), which permits any noncommercial use, distribution, and reproduction in any medium, provided you give appropriate credit to the original author(s) and the source, provide a link to the Creative Commons license, and indicate if changes were made.

\section{References}

1. Chen W, Zheng R, Baade PD, Zhang S, Zeng H, Bray F, et al. Cancer statistics in China, 2015. CA Cancer J Clin. 2016;66(2):11532. https://doi.org/10.3322/caac.21338.

2. Kilgoz HO, Bender G, Scandura JM, Viale A, Taneri B. Kras and the reality of personalized medicine in non-small cell lung cancer. Mol Med. 2016;22:380-7. https://doi.org/10.2119/molme d.2016.00151.

3. Novello S, Barlesi F, Califano R, Cufer T, Ekman S, Levra MG, et al. Metastatic non-small-cell lung cancer: ESMO Clinical Practice Guidelines for diagnosis, treatment and follow-up. Ann Oncol. 2016;27(suppl 5):v1-27. https://doi.org/10.1093/annonc/ mdw326.

4. Oser MG, Niederst MJ, Sequist LV, Engelman JA. Transformation from non-small-cell lung cancer to small-cell lung cancer: molecular drivers and cells of origin. Lancet Oncol. 2015;16(4):e16572. https://doi.org/10.1016/S1470-2045(14)71180-5.

5. William WN Jr, Lin HY, Lee JJ, Lippman SM, Roth JA, Kim ES. Revisiting stage IIIB and IV non-small cell lung cancer: analysis of the surveillance, epidemiology, and end results data. Chest. 2009;136(3):701-9. https://doi.org/10.1378/chest.08-2968.

6. Rutkowski J, Saad ED, Burzykowski T, Buyse M, Jassem J. Chronological trends in progression-free, overall, and postprogression survival in first-line therapy for advanced NSCLC. J Thorac Oncol. 2019. https://doi.org/10.1016/j.jtho.2019.05.030.

7. Valentino F, Borra G, Allione P, Rossi L. Emerging targets in advanced non-small-cell lung cancer. Future Oncol. 2018;14(13s):61-72. https://doi.org/10.2217/fon-2018-0099.

8. Reck M, Garassino MC, Imbimbo M, Shepherd FA, Socinski MA, Shih JY, et al. Antiangiogenic therapy for patients with aggressive or refractory advanced non-small cell lung cancer in the second-line setting. Lung Cancer. 2018;120:62-9. https://doi. org/10.1016/j.lungcan.2018.03.025

9. Shafique M, Tanvetyanon T. Immunotherapy alone or chemoimmunotherapy as front-line treatment for advanced non-small cell lung cancer. Expert Opin Biol Ther. 2019;19(3):225-32. https ://doi.org/10.1080/14712598.2019.1571036. 
10. Cancer Care Ontario (Lung Cancer Disease Site Group) (2016) Evidence-based Series and Practice Guidelines: Systemic Treatment for Patients with Advanced Non-Small Cell Lung Cancer. https://www.cancercare.on.ca/common/pages/UserFile.aspx?fileI d=366079. Accessed 11 Dec 2017.

11. Doroshow DB, Sanmamed MF, Hastings K, Politi K, Rimm DL, Chen L, et al. Immunotherapy in non-small cell lung cancer: facts and hopes. Clin Cancer Res. 2019;25(15):4592-602. https://doi. org/10.1158/1078-0432.CCR-18-1538.

12. Vokes EE, Ready N, Felip E, Horn L, Burgio MA, Antonia SJ, et al. Nivolumab versus docetaxel in previously treated advanced non-small-cell lung cancer (CheckMate 017 and CheckMate 057): 3 -year update and outcomes in patients with liver metastases. Ann Oncol. 2018;29(4):959-65. https://doi.org/10.1093/annonc/mdy04 1.

13. Gettinger S, Horn L, Jackman D, Spigel D, Antonia S, Hellmann $\mathrm{M}$, et al. Five-year follow-up of nivolumab in previously treated advanced non-small-cell lung cancer: results from the CA209003 study. J Clin Oncol. 2018;36(17):1675-84. https://doi. org/10.1200/JCO.2017.77.0412.

14. Wu YL, Lu S, Cheng Y, Zhou C, Wang J, Mok T, et al. Nivolumab versus docetaxel in a predominantly chinese patient population with previously treated advanced NSCLC: CheckMate 078 randomized phase III clinical trial. J Thorac Oncol. 2019;14(5):86775. https://doi.org/10.1016/j.jtho.2019.01.006.

15. Chinese Society of Clinical Oncology (CSCO). Primary lung cancer treatment guidelines (2018 version). http://guide.medlive.cn/ guideline/16783. Accessed 23 Jul 2018.

16. National Bureau of Statistics of China: China statistical year book 2018. http://data.stats.gov.cn/english/index.htm. Accessed $20 \mathrm{Feb}$ 2019.

17. China Center for Health Economic Research. China guidelines for pharmacoeconomic evaluations (Version 2011) [in Chinese]. https://tools.ispor.org/peguidelines/. Accessed 12 Oct 2019.

18. Hoyle MW, Henley W. Improved curve fits to summary survival data: application to economic evaluation of health technologies. BMC Med Res Methodol. 2011;11:139. https://doi. org/10.1186/1471-2288-11-139.

19. Zeng X, Li J, Peng L, Wang Y, Tan C, Chen G, et al. Economic outcomes of maintenance gefitinib for locally advanced/metastatic non-small-cell lung cancer with unknown EGFR mutations: a semi-Markov model analysis. PLoS One. 2014;9(2):e88881. https ://doi.org/10.1371/journal.pone.0088881.

20. Nafees B, Lloyd AJ, Dewilde S, Rajan N, Lorenzo M. Health state utilities in non-small cell lung cancer: an international study. Asia Pac J Clin Oncol. 2017;13(5):e195-203. https://doi.org/10.1111/ ajco.12477.

21. Lu S, Yu Y, Fu S, Ren H. Cost-effectiveness of ALK testing and first-line crizotinib therapy for non-small-cell lung cancer in China. PLoS One. 2018;13(10):e0205827. https://doi. org/10.1371/journal.pone.0205827.

22. Guan H, Sheng Y, Guo W, Han S, Shi L. Cost-effectiveness of alectinib for patients with untreated ALK-positive non-small cell lung cancer in China. Adv Ther. 2019;36(5):1114-25. https://doi. org/10.1007/s12325-019-00908-7.
23. Wu B, Dong B, Xu Y, Zhang Q, Shen J, Chen H, et al. Economic evaluation of first-line treatments for metastatic renal cell carcinoma: a cost-effectiveness analysis in a health resource-limited setting. PLoS One. 2012;7(3):e32530. https://doi.org/10.1371/ journal.pone.0032530.

24. Zhang C, Zhang H, Shi J, Wang D, Zhang X, Yang J, et al. Trialbased cost-utility analysis of icotinib versus Gefitinib as secondline therapy for advanced non-small cell lung cancer in China. PLoS One. 2016;11(3):e0151846. https://doi.org/10.1371/journ al.pone.0151846.

25. Zeng X, Karnon J, Wang S, Wu B, Wan X, Peng L. The cost of treating advanced non-small cell lung cancer: estimates from the chinese experience. PLoS One. 2012;7(10):e48323. https://doi. org/10.1371/journal.pone.0048323.

26. Wu B, Li T, Cai J, Xu Y, Zhao G. Cost-effectiveness analysis of adjuvant chemotherapies in patients presenting with gastric cancer after D2 gastrectomy. BMC Cancer. 2014;14:984. https:// doi.org/10.1186/1471-2407-14-984.

27. Gordon L. China guidelines for pharmacoeconomic evaluation and manual. 2015th ed. Beijing: Science Press; 2015.

28. Carrera PM, Kantarjian HM, Blinder VS. The financial burden and distress of patients with cancer: understanding and stepping-up action on the financial toxicity of cancer treatment. CA Cancer J Clin. 2018;68(2):153-65. https://doi.org/10.3322/caac.21443.

29. Zhu J, Li T, Wang X, Ye M, Cai J, Xu Y, et al. Gene-guided gefitinib switch maintenance therapy for patients with advanced EGFR mutation-positive non-small cell lung cancer: an economic analysis. BMC Cancer. 2013;13:39. https://doi. org/10.1186/1471-2407-13-39.

30. Wan X, Zhang Y, Tan C, Zeng X, Peng L. First-line nivolumab plus ipilimumab vs sunitinib for metastatic renal cell carcinoma: a cost-effectiveness analysis. JAMA Oncol. 2019;5(4):491-6. https ://doi.org/10.1001/jamaoncol.2018.7086.

31. Gao L, Li SC. Modelled economic evaluation of nivolumab for the treatment of second-line advanced or metastatic squamous non-small-cell lung cancer in australia using both partition survival and Markov models. Appl Health Econ Health Policy. 2019;17(3):371-80. https://doi.org/10.1007/s40258-018-0452-0.

32. Shafrin J, Skornicki M, Brauer M, Villeneuve J, Lees M, Hertel $\mathrm{N}$, et al. An exploratory case study of the impact of expanding cost-effectiveness analysis for second-line nivolumab for patients with squamous non-small cell lung cancer in Canada: does it make a difference? Health Policy. 2018;122(6):607-13. https://doi. org/10.1016/j.healthpol.2018.04.008.

33. Hu S, Fan L, Tang Z, Harrison JP, Hertel $\mathrm{N}$ et al. An economic evaluation of nivolumab for the treatment of advanced non-small cell lung cancer (ANSCLC) after prior chemotherapy in china. Value Health. 2019.

34. Fehrenbacher L, Spira A, Ballinger M, Kowanetz M, Vansteenkiste J, Mazieres J, et al. Atezolizumab versus docetaxel for patients with previously treated non-small-cell lung cancer (POPLAR): a multicentre, open-label, phase 2 randomised controlled trial. Lancet. 2016;387(10030):1837-46. https://doi.org/10.1016/S0140 $-6736(16) 00587-0$. 